\title{
Influences combinées d'injections de 1-29 GRF et du niveau alimentaire énergétique chez la chèvre en lactation
}

\author{
D Sauvant1', G Kann², J Hervieu'1, N Mandran'1, C Disenhaus'1 \\ ${ }^{1}$ Station de Nutrition et Alimentation (INRA) de I'INA-PG, 16, rue C-Bernard, 75231 Paris Cedex 05; \\ 2 Laboratoire de Physiologie et de la Lactation (INRA), \\ CR Domaine de Vilvert, 78350 Jouy-en-Josas, France
}

Summary - Subcutaneous injections of 1-29 GRF and diet energy level were studied in 48 dairy goats using a $2 \times 2$ factorial design. Energy and GRF effects were additive on milk production and some interactions were observed on the milk fat and protein contents. Subcutaneous injections seemed to be less efficient in promoting milk secretion than the intravenous route used in a previous trial.

Introduction - Une étude précédente a montré que des injections de 1-29 GRF augmentaient la production laitière de la chèvre (Sauvant et al, 1988). Une expérimentation a été entreprise pour tester les interactions entre l'apport de GRF et le niveau énergétique de la ration.

Matériel et Méthodes - Quarante huit chèvres, Alpines ou Saanen, placées en cages individuelles, ont été réparties en 4 lots égaux pour tester, selon un plan factoriel $2 \times 2$, les effets et l'interaction entre, d'une part, des injections sous-cutanées biquotidiennes de GRF 129 (produit fourni par Sanofi) à la dose de 0,76 $\mathrm{nmol} / \mathrm{kg}$ de poids vif et, d'autre part, le niveau d'apport d'énergie. Les animaux des 2 lots bas en énergie (B) subissent en début de période expérimentale une baisse de $200 \mathrm{~g}$ de MS de l'apport de concentré, puis des baisses successives de $70 \mathrm{~g} \mathrm{MS} / 2$ semaines. Les animaux des lots haut en énergie $(H)$ ne subissent pas de baisse initiale d'apport concentré et les réductions de l'apport dans le temps ne sont que de $50 \mathrm{~g} \mathrm{MS} / 2$ semaines. Le reste de la ration, du foin de luzerne et de la pulpe de betterave ensilée, est distribué ad libitum dans les lots $\mathrm{H}$ et en quantité limitée pour les lots $B$.

La période expérimentale dure 12 semaines, précédées par une prépériode de 4 semaines.
Les chèvres sont entre leur $4^{\theta}$ et $8^{\theta}$ semaine de lactation au début de la période expérimentale. Toutes les 3 semaines, des prélèvements de sang ont été effectués en vue du dosage du glucose et des acides gras non estérifiés (AGNE) et de l'urée qui a également été dosée dans le lait. Pour évaluer l'évolution des effets, l'interprétation statistique est effectuée par analyse de covariance, d'une façon séparée pour les 4 sous-périodes expérimentales de 3 semaines. La parité et le type de caséine $\alpha S 1$ ont été pris en compte en facteur bloc dans l'interprétation.

Résultats et Discussion - Les niveaux d'ingestion de matière sèche (tableau I) et d'énergie sont significativement plus élevés dans le lot $H$ et ne sont pas influencés par le GRF. La production de lait brut est plus importante $(P<0,01)$ pour le lot $\mathrm{H}$; elle est également plus élevée pour les animaux injectés au GRF aux périodes 2, 3 et 4; il n'y a pas d'interaction entre les 2 facteurs. L'effet du GRF sur la production est nettement moins important que dans l'étude précédente (Sauvant et al, 1988): $+22 \mathrm{~g} / \mathrm{j} v s+285 \mathrm{~g} / \mathrm{j}$, peut-être en raison de la différence de modalité d'injection (sous- 
Tableau I. Evolution des principales caractéristiques zootechniques pendant les 4 périodes de mesure.

\begin{tabular}{|c|c|c|c|c|c|}
\hline & & 1 & 2 & 3 & 4 \\
\hline $\begin{array}{l}\text { Matière sèche } \\
\text { ingérée }(g / j)\end{array}$ & $\begin{array}{l}m \\
\mathrm{E} \\
\mathrm{G}\end{array}$ & $\begin{array}{c}2238 \\
244 \text { ** } \\
18\end{array}$ & $\begin{array}{c}2186 \\
282 \text { ** } \\
6\end{array}$ & $\begin{array}{c}2110 \\
350^{* *} \\
48\end{array}$ & $\begin{array}{c}2007 \\
388 \text { ** } \\
44\end{array}$ \\
\hline $\begin{array}{l}\text { Production de } \\
\text { lait brut }(g / j)\end{array}$ & $\begin{array}{l}m \\
E \\
G\end{array}$ & $\begin{array}{c}2825 \\
38 \text { ** } \\
2\end{array}$ & $\begin{array}{r}2815 \\
35 \text { ** } \\
24 \text { * }\end{array}$ & $\begin{array}{r}2793 \\
55 * * \\
36 * *\end{array}$ & $\begin{array}{r}2755 \\
46 \text { ** } \\
24 \text { " }\end{array}$ \\
\hline $\begin{array}{l}\text { Taux butyreux } \\
(\mathbf{g} / \mathbf{l})\end{array}$ & $\begin{array}{l}m \\
\mathrm{E} \\
\mathrm{G}\end{array}$ & $\begin{array}{r}35,2 \\
0,4 \\
1,6\end{array}$ & $\begin{array}{r}34,3 \\
-1,0 \\
1,2\end{array}$ & $\begin{array}{r}35,0 \\
-0,7 \\
1,0\end{array}$ & $\begin{array}{r}33,8 \\
-1,6 \\
-1,0\end{array}$ \\
\hline $\begin{array}{l}\text { Taux protéique } \\
(\mathrm{g} / \mathrm{l})\end{array}$ & $\begin{array}{l}m \\
\mathrm{E} \\
\mathrm{G}\end{array}$ & $\begin{array}{c}26,9 \\
0,5 \\
0,0\end{array}$ & $\begin{array}{r}26,6 \\
0,1 \\
-0,2\end{array}$ & $\begin{array}{r}26,8 \\
-0,4 \\
-0,2\end{array}$ & $\begin{array}{r}26,9 \\
0,2 \\
-0,9\end{array}$ \\
\hline
\end{tabular}

$m$ : moyenne des 48 chèvres; $E$ : effet du niveau d'énergie; ** significatif à $P<0,01$; $G$ : effet de l'apport de GRF; * significatif a $P<0,05$.

cutané vs intraveineux). Le taux butyreux du lait n'est pas modifié par les 2 facteurs qui présentent par contre une interaction significative aux périodes 2 et 3 . Le taux protéique (TP) est accru de 0,5 point dans les lots $\mathrm{H}$ en première période $(P<0,05)$; en outre une interaction apparaît entre les 2 facteurs aux périodes $1(P<0,01)$ et 2 $(P<0,05)$ car l'accroissement du TP des animaux au régime $H$ ne s'observe que dans le cas où il n'y a pas de GRF injecté. Le bilan moyen d'énergie calculé est plus important $(+126 \mathrm{kcal} / \mathrm{j}, P<0,01)$ pour le lot $H$ mais n'est pas modifié par l'apport de GRF. Les chèvres du régime $B$ perdent légèrement du poids, $-70 \mathrm{~g} / \mathrm{semaine}$, alors que celles des lots $\mathrm{H}$ ont pris en moyenne $167 \mathrm{~g} / \mathrm{semaine}$ (différence significative à $P<0,01$ ); les apports de GRF n'ont pas influencé l'évolution pondérale.
Les teneurs en nutriments énergétiques du plasma, glucose et AGNE, ne sont pas modifiées par les apports d'énergie et de GRF en raison du faible écart des valeurs des bilans énergétiques. Les teneurs en urée du plasma et du lait ne sont également pas modifiées par les facteurs expérimentaux.

En conclusion, les injections souscutanées de GRF 1-29 sont apparues moins efficaces que par la voie intraveineuse pour accroître la production laitière. Mis á part les taux butyreux et protéiques de certaines périodes, il n'y pas eu d'interaction entre les effets du GRF et du niveau d'apport d'énergie.

Sauvant D, Kann G, Hervieu J, Disenhaus C (1988) Reprod Nutr Dev 28 (suppl 1), 179180 